\title{
Modélisation thermodynamique du comportement cyclique d'alliages à mémoire de forme TiNi et CuZnAl sollicités en traction ondulée
}

\section{G. BOURBON et C. LEXCELLENT}

Laboratoire de Mécanique Appliquée R. Chaléat, Associé au CNRS, UFR Sciences et Techniques La Bouloie, Route de Gray, 25030 Besançon cedex, France

\section{RESUME}

L'extension au cas cyclique, d'un modèle de comportement pseudoélastique d'alliages à mémoire de forme (A.M.F.), mis au point par Raniecki, Lexcellent et Tanaka, ne pose pas de problème. La forme des boucles d'hystérésis permet une identification plus fiable des paramètres thermodynamiques pour le $\mathrm{Ti} \mathrm{Ni}$ que pour le $\mathrm{Cu} \mathrm{Zn} \mathrm{Al}$.

\section{ABSTRACT}

The extension for the cyclic case, of the shape memory alloys (S.M.A.) pseudoelastic behavior modelling, written by Raniecki, Lexcellent and Tanaka, is done without problem. The shape of hysteresis loops allows a more accurate identification of thermodynamical parameters for $\mathrm{Ti} \mathrm{Ni}$ than for $\mathrm{Cu} \mathrm{Zn} \mathrm{Al}$.

\section{1 - INTRODUCTION}

La modélisation thermodynamique du comportement pseudoélastique d'alliages à mémoire de forme (A.M.F.) sollicités en traction isotherme $\left(T>A_{F}: A_{F}\right.$ température de fin d'apparition de l'austénite) a été effectuée. Dans un cadre "idéal", Müller et Xu [1] ont modélisé le comportement de monocristaux de $\mathrm{Cu} \mathrm{Zn} \mathrm{Al}$. Raniecki et Lexcellent [2] ont étendu la modélisation aux polycristaux de $\mathrm{Ti} \mathrm{Ni}$ et de $\mathrm{Cu} \mathrm{Zn} \mathrm{Al}$. Dans les deux approches, l'hystérésis a été prise en compte par l'introduction dans l'énergie libre globale d'un terme de cohésion entre les deux phases (mère : austenite - produite : martensite). La modélisation cyclique consiste en une extension du modèle pseudoélastique mis au point par Raniecki, Lexcellent et Tanaka [3]. A ce jour, à notre connaissance, seule une approche phénoménologique des lois de comportement cyclique a été effectuée [4].

Dans cette publication, nous présentons :

(i) Les résultats expérimentaux relatant le comportement cyclique isotherme du Ti Ni et du Cu Zn Al 
(ii) Le modèle thermodynamique du comportement cyclique $\left(T>A_{F}\right)$

(iii) L'identification des paramètres et la simulation des courbes expérimentales

\section{2 - ESSAIS CYCLIQUES ISOTHERMES SUR LE Ti Ni etle Cu Zn Al}

\subsection{Essais sur le Ti Ni}

Des tests de traction ondulée sur des fils de Ti-55,3\% Pd.Ni ont été réalisés par Tobushi et col. [5] à $\mathrm{T}=333,353,373 \mathrm{~K}\left(\mathrm{~T}>\mathrm{A}_{\mathrm{F}}=321 \mathrm{~K}\right) ; \dot{\varepsilon}=6,67.10^{-4} \mathrm{~S}^{-1}$ nombre de cycles $\mathrm{N}=100$, cyclage entre $\sigma=0$ et $\varepsilon_{\max }=0,08$.

La courbe expérimentale $(\mathrm{T}=373 \mathrm{~K}$, fig. 1$)$ montre que :

-Le premier demi-cycle $(N=1)$ est pratiquement pseudoélastique avec une très forte hystérésis.

- Au cours du cyclage ( $N$ croissant), la déformation résiduelle $\varepsilon_{i r}$ obtenue à contrainte nulle $(\sigma=0)$, augmente et tend vers une valeur asymptotique $\varepsilon_{i r}$ correspondant à une stabilisation du cycle.

- Les contraintes seuils de transformation de phase $\sigma \mathrm{AM}(\mathrm{N})$ et $\sigma \mathrm{MA}(\mathrm{N})$ diminuent avec N jusqu'à une valeur asymptotique $\sigma \mathrm{AM}(\infty)$ et $\sigma \mathrm{MA}(\infty)$.

\subsection{Essais sur le $\mathrm{Cu} \mathrm{Zn} \mathrm{Al}$}

Les résultats expérimentaux obtenus sur des polycristaux de Cu-25,63\% $\mathrm{Zn}, 4,2 \% \mathrm{Al}$ (Pds) sont plus difficilement exploitables (fig. 2). Température d'essai $296 \mathrm{~K}>\mathrm{A}_{\mathrm{F}}=292 \mathrm{~K}$. $\varepsilon=10^{-3} \mathrm{~s}^{-1}$, cyclage entre $\sigma=0$ et $\varepsilon_{\max }=1 \%$. Traitement thermique $300 \mathrm{~s}$ à $1123 \mathrm{~K}$ suivi d'une trempe à l'huile à $393 \mathrm{~K}$ pendant $3600 \mathrm{~s}$. Seuls les seuils oAM(N) sont mesurables et diminuent avec $\mathrm{N}\left(\mathrm{N}_{\max }=22\right)$. Des mesures de résistance électrique réalisées "in situ" pendant l'essai de traction ondulée, permettent de dire que la déformation $\varepsilon_{i r}(N)$ à $\sigma=0$ (qui augmente avec $\mathrm{N}$ ) correspond à de la martensite résiduelle.

\section{3 - MODELE THERMODYNAMIQUE DE COMPORTEMENT CYCLIQUE ISO-}

\section{THERME DES A.M.F. (a T $\left.>A_{F}\right)$}

\subsection{Expresion de l'énergie libre}

L'extension au chargement cyclique du modèle pseudoélastique explicité en [3], donne l'expression de l'énergie libre spécifique à l'état d"équilibre contraint" (concept de Kestin et Rice [6]).

$$
\begin{gathered}
\Phi_{c}(\varepsilon, T, z, h)=\frac{E}{2 \rho}\left(\varepsilon-y^{z}-\varepsilon_{T}\right)^{2}+\int_{T_{0}}^{T}\left(\frac{T_{1}-T}{T_{1}}\right) C_{\sigma}\left(T_{1}\right) d T \\
+z(1-z) \Phi_{i t}(T)+u_{0}^{*(1)}-T_{0}^{*}(2)-z \Pi_{0}^{f}(T)+\Phi_{t r}(h, T, z)
\end{gathered}
$$

avec $a=1:$ austenite $; \alpha=2:$ martensite 
* $\mathrm{z}$ : fraction massique de martensite totale, $\mathrm{Y}$ : déformation de transformation complète de phase

${ }^{*} \mathrm{~h}=\mathrm{z}_{\mathrm{ir}}$ : fraction de martensite résiduelle (qui s'accumule au cours du cyclage donc quiest non restaurable sauf par traitement thermique)

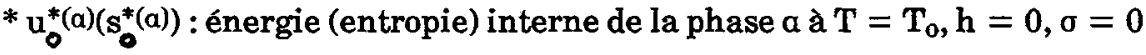

$$
\mathrm{n}_{0}^{\mathrm{f}}(\mathrm{T})=\Delta \mathrm{u}^{*}-\mathrm{T} \Delta \mathrm{s}^{*}\left(\Delta \mathrm{u}^{*}=\mathrm{u}_{0}^{*(1)}-\mathrm{u}_{0}^{*(2)}, \Delta \mathrm{s}^{*}=\mathrm{s}_{0}^{*(1)}-\mathrm{s}_{0}^{*(2)}\right)
$$

* E,,, $\mathrm{C}_{0}(\mathrm{~T})$ : module d'Young, masse volumique, chaleur spécifique à contrainte constante (les mêmes pour les deux phases)

${ }^{*} \varepsilon_{T}=a\left(T-T_{0}\right):$ terme de dilation thermique

${ }^{*} \mathrm{z}(1-\mathrm{z}) \Phi_{\mathrm{it}}(\mathrm{T})$ avec $\Phi_{\mathrm{it}}(\mathrm{T})=\overline{\mathrm{u}}_{0}-\mathrm{T} \overline{\mathrm{s}}_{0}:$ terme de cohésion entre les deux phases

* $\Phi_{\mathrm{tr}}(\mathrm{h}, \mathrm{T}, \mathrm{z})$ : terme de la contribution cyclique à l'énergie libre

$$
\begin{aligned}
& \Phi_{\mathrm{tr}}(\mathrm{h}, \mathrm{T}, \mathrm{z})=\Phi^{*(1)}(\mathrm{h}, \mathrm{T})-\mathrm{z} \Delta \mathrm{r}_{\mathrm{o}}^{\mathrm{f}}(\mathrm{h}, \mathrm{T}) \\
& \Phi_{\mathrm{h}}^{*(1)}(\mathrm{h})=\mathrm{u}_{\mathrm{h}}^{*(1)}(\mathrm{h})-\mathrm{Ts}_{\mathrm{h}}^{*(1)}(\mathrm{h}) \\
& \Delta \mathrm{r}_{0}^{\mathrm{f}}(\mathrm{h}, \mathrm{T})=\Delta \mathrm{u}_{\mathrm{h}}^{*}(\mathrm{~h})-\mathrm{T} \Delta \mathrm{s}_{\mathrm{h}}^{*}(\mathrm{~h})\left(\Delta \mathrm{u}_{\mathrm{h}}^{*}=\mathrm{u}_{\mathrm{h}}^{*(1)}(\mathrm{h})-\mathrm{u}_{\mathrm{h}}^{*(2)}(\mathrm{h}), \Delta \mathrm{s}_{\mathrm{h}}^{*}=\mathrm{s}_{\mathrm{h}}^{*(1)}-\mathrm{s}_{\mathrm{h}}^{*(2)}\right)
\end{aligned}
$$

\subsection{Equations d'état}

$$
\left\{\begin{array}{l}
\sigma=\rho \frac{\partial \Phi_{c}}{\partial \varepsilon}=E\left(\varepsilon-\mathrm{Yz}-\varepsilon_{\mathrm{T}}\right) \\
\mathrm{s}=-\frac{\partial \Phi_{\mathrm{c}}}{\partial T}
\end{array}\right.
$$

Aux deux paramètres $\mathrm{z}$ et $\mathrm{h}$, sont associées les forces thermodynamiques $\Pi^{f}$ et $\Pi^{h}$ :

$$
\left\{\begin{array}{l}
\mathrm{n}^{\mathrm{f}}=-\frac{\partial \Phi_{c}}{\partial z}=\Pi_{0}^{f}(T)-(1-2 z) \Phi_{i t}(T)+\frac{\sigma \Psi}{\rho}+\Delta n_{0}^{f}(h, T) \\
n^{h}=-\frac{\partial \Phi_{c}}{\partial h}=z \frac{\partial}{\partial h}\left(\Delta \Pi_{0}^{f}(h, T)\right)-\frac{\partial \Phi_{h}^{(1)}}{\partial h}
\end{array}\right.
$$

L'inégalité de Clausius Duhem s'écrit:

$$
d D=\pi^{f} d z+\pi^{h} d h \geq 0
$$

Comme nous allons choisir $\pi^{\mathrm{h}} \geq 0$ ainsi que $\mathrm{dh} \geq 0$, l'inégalité de Clausius Duhem sera toujours respectée si $\mathrm{n}^{f} \mathrm{dz} \geq 0$.

Ainsi les lignes d"équilibre instable" [1],[3] seront définies par $\mathrm{n}^{f}=\mathbf{0}$. 


\subsection{Equations cinétiques de $z$ et $h$}

Comme en "plasticité", nous supposerons qu'il existe deux fonctions critères $\Psi^{(a)}\left(\mathbf{n}^{f}, \mathbf{z}, \mathbf{h}\right)$ $(\alpha=1,2)$ telles qu'un processus actif de décomposition de la phase mère $(A \rightarrow M: d z>0)$ ne puisse avoir lieu que si $\Psi^{(1)}=$ constante $\left(d \Psi^{(1)}=0\right)$. La transformation inverse $(M \rightarrow A)$ correspondant à $\Psi^{(2)}=\mathrm{Cte}\left(\mathrm{d} \Psi^{(2)}=0\right)$. Les boucles externes pour la transformation complète correspondent à $\Psi^{(a)}=0$, les boucles internes correspondant à une transformation partielle sont régies par $\Psi^{(a)}=$ Cte $<0$. Une extension de [3] au cas cyclique donne

$$
\Psi^{(1)}=\Pi^{f}-k^{(1)}(z, h), \Psi^{(2)}=-\Pi^{f}+k^{(2)}(z, h)
$$

avec

$$
\left\{\begin{array}{l}
k^{(1)}(z, h)=-\left(A_{1}+B_{1}\left(\frac{z-h}{1-h}\right)\right) \operatorname{Ln}\left(\frac{1-z}{1-h}\right)+C_{1}\left(\frac{z-h}{1-h}\right) \\
k^{(2)}(z, h)=\left(A_{2}-B_{2}\left(\frac{1-z}{1-h}\right)\right) \operatorname{Ln}\left(\frac{z-h}{1-h}\right)-C_{2}\left(\frac{1-z}{1-h}\right)
\end{array}\right.
$$

avec :

$$
\begin{gathered}
a_{1} A_{1}=\Delta s^{*}-\bar{s}_{0}, a_{2} A_{2}=\Delta s^{*}+\bar{s}_{0} \\
a_{1} B_{1}=a_{2} B_{2}=2 \bar{s}_{0} \\
C_{1}=2 \Phi_{i t}\left(M_{s}^{9}\right), C_{2}=2 \Phi_{i t}\left(A_{s}^{9}\right)
\end{gathered}
$$

Enfin, une formulation simple de la cinétique de h peut être :

$$
\dot{h}=m_{0}\left(h_{\infty}-h\right)^{p}<\dot{z}><\dot{z}>=\left\{\begin{array}{l}
\dot{z} \text { si } \dot{z}>0 \\
0 \text { si } \dot{z}<0
\end{array}\right.
$$

avec $\mathrm{m}_{0}, \mathrm{~h}_{\infty}, \mathrm{p}$ sont des constantes à température fixée.

\section{4 - IDENTIFICATION DES CONSTANTES DU MODELE CYCLIQUE ET SIMU- LATION}

La modélisation du premier demi-cycle $(\mathrm{N}=1)$ qui est considéré comme pseudoélastique [2] donne les paramètres $\rho, E, \Delta u^{*}, \bar{u}_{0}, \Delta s^{*}, \bar{s}_{0}, y, a_{1}, a_{2}$. Il reste à déterminer $\Delta \mathrm{n}^{\mathrm{f}}(\mathrm{h}, \mathrm{T})\left(\Delta \mathrm{u}^{*}(\mathrm{~h})\right.$, $\left.\Delta \mathrm{s}^{*}(\mathrm{~h})\right), \mathrm{m}_{0}, \mathrm{p}, \mathrm{h}_{\infty}$.

L'évolution du seuil $\sigma \mathrm{AM}(\mathrm{N})$ avec $\mathrm{N}$ permet d'écrire :

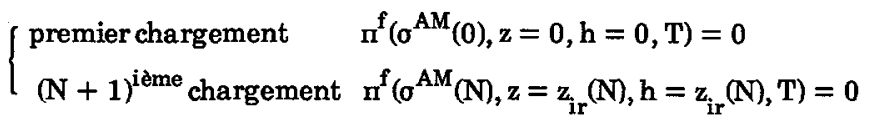

$$
\begin{aligned}
& \Rightarrow \frac{\left(\sigma^{A M}(0)-\sigma^{A M}(N)\right) Y}{\rho}=2 z_{i r}(N) \Phi_{i t}(T)+\Delta r_{0}^{f}\left(h(N)=z_{i r}(N), T\right)
\end{aligned}
$$

avec $\varepsilon_{i r}(\mathrm{~N})$ mesurable à la fin de chaque cycle $(\sigma=0)$

$$
\varepsilon_{\mathrm{ir}}(\mathrm{N})=\mathrm{Y}_{\mathrm{ir}}(\mathrm{N})
$$

$\Rightarrow \Delta \Pi^{f}(h(N, T))$ identifiable à chaque cycle (équation 13) est écrite sous forme polynomiale (fig. 3 : Ti Ni) 


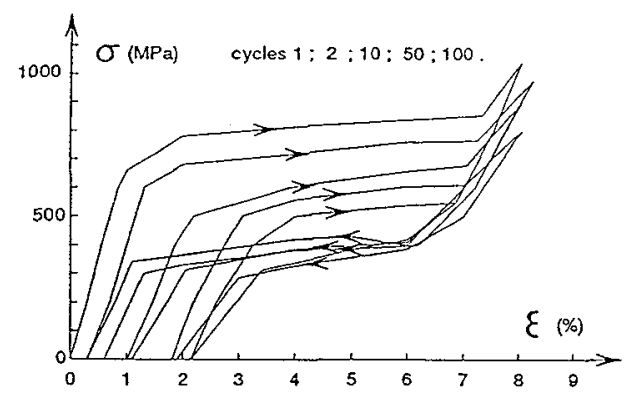

Fig 1 : Ti Ni

Traction ondulée cyclique

Courbe experimentale $(T=373 \mathrm{~K}$ )

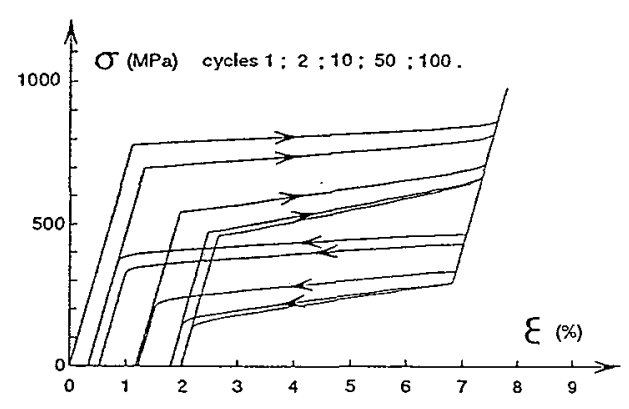

Fig 5: Ti Ni

Traction ondulée cyclique

Courbe simulée $(T=373 \mathrm{~K})$

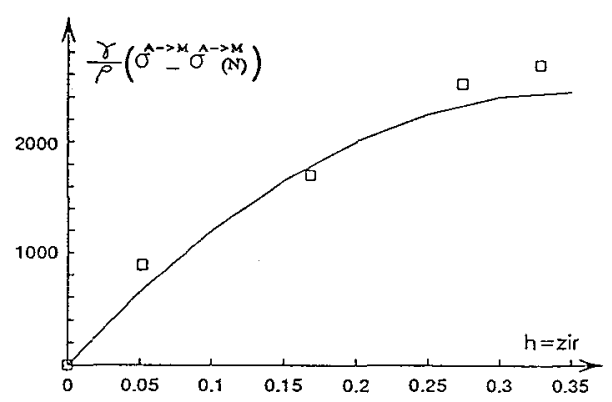

Fig 3 : Ti Ni

Détermination experimentale de $\bar{\phi}$ et $\bar{\phi}$

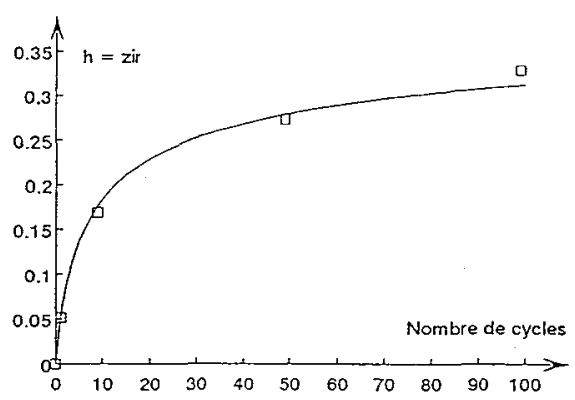

Fig 4 : Ti Ni

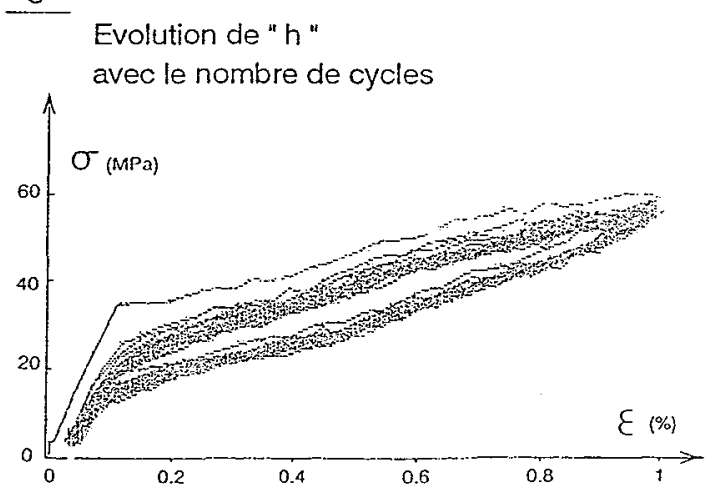

Fig 2: Cu Zn Al

Traction ondulée cyclique

Courbe experimentale $(\mathrm{T}=296 \mathrm{~K})$

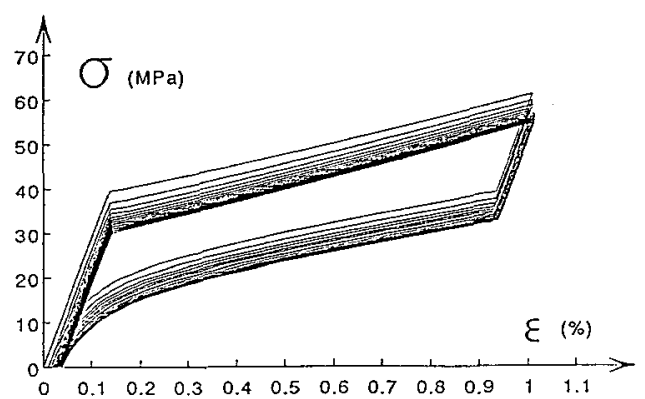

Fig 6: Cu Zn Al

Traction ondulée cyclique

Courbe simulée $(T=296 \mathrm{~K})$ 


$$
\Delta_{\mathbf{n}_{0}}^{f}(h, T)=-\frac{1}{2} h^{2} \bar{\Phi}(T)+h \overline{\bar{\Phi}}(T)
$$

Enfin, l'évolution de

$$
\mathrm{k}(\mathrm{N}, \sigma=0)=z_{\mathrm{ir}}(\mathrm{N}, \sigma=0)\left(=\frac{\varepsilon_{\mathrm{ir}}(\mathrm{N}, \sigma=0)}{\mathrm{Y}}\right)
$$

avec $\mathrm{N}$ permet d'obtenir $\mathrm{m}_{0}, \mathrm{p}, \mathrm{h}^{\infty}$. (fig. 4 : Ti $\mathrm{Ni}$ )

\begin{tabular}{|c|c|c|c|c|c|c|c|c|}
\hline Alliage & $\underset{N / m^{2}}{E}$ & $\underset{\mathrm{kg} / \mathrm{m} 3}{\rho}$ & $\underset{\mathbf{K}}{\mathbf{M}_{\mathbf{S}}}$ & $\frac{\mathrm{A}_{\mathrm{s}}}{\mathrm{K}}$ & $\begin{array}{l}\Delta u^{*} \\
\mathbf{J} / \mathbf{K g}\end{array}$ & $\underset{\mathrm{J} / \mathrm{Kg}}{\bar{u}_{0}}$ & $\begin{array}{c}\Delta \mathrm{s}^{*} \\
\mathrm{~J} / \mathrm{Kg} \mathrm{K}\end{array}$ & $\begin{array}{c}\overline{\mathrm{s}}_{\mathrm{o}} \\
\mathrm{J} / \mathrm{K} \mathrm{g} K\end{array}$ \\
\hline $\mathrm{Ti} \mathrm{Ni}$ & 71010 & 6500 & 230 & 305 & 17400 & 2500 & 64 & 3,5 \\
\hline $\mathrm{Cu} \mathrm{Zn} \mathrm{Al}$ & $2,910^{10}$ & 7700 & 287 & 290 & 10000 & 6000 & 34,4 & 20,2 \\
\hline Alliage & $\underset{\mathrm{J} / \mathrm{Kg}}{\Phi}$ & $\underset{\mathrm{J} / \mathrm{Kg}}{\Phi}$ & $\mathrm{p}$ & $\mathrm{m}_{0}$ & $h_{\infty}$ & $\stackrel{\mathrm{a}_{1}}{\mathrm{~K}-1}$ & $\underset{\mathrm{K}-1}{\mathrm{a}}$ & \\
\hline $\mathrm{TiNi}$ & 40000 & 14000 & 3,9 & 1 & 0,44 & 1,2 & 1,2 & \\
\hline $\mathrm{Cu} \mathrm{Zn} \mathrm{Al}$ & 40000 & 5000 & 2,45 & 800 & 0,012 & 0,25 & 0,8 & \\
\hline
\end{tabular}

Les constantes nécessaires à la simulation cyclique sont regroupées dans le tableau suivant.

La simulation des courbes expérimentales données en figures 1 et 2 est correcte (Ti $\mathrm{Ni}$, Fig. 5) (Cu Zn Al, Fig. 6).

\section{CONCLUSION}

L'extension au cas cyclique d'un modèle isotherme de comportement pseudoélastique des A.M.F. ne pose pas de problème majeur. L'étape suivante consistera en la modélisation de la boucle (déformation $\leftrightarrow$ température) obtenue à l'état libre de contrainte après ce processus d'éducation ( $\mathrm{N}$ demi-cycles de traction ondulée). Par contre, la modélisation du comportement cyclique anisotherme (de part et d'autre des températures de transformation), sous sollicitation mécanique constante, s'avère nettement plus délicate.

\section{REFERENCES}

[1] Müller I. et Xu H., Acta Met. Mater. 29, 3 (1991) 263

[2] Raniecki B. et Lexcellent C., Eur. J. Mech. Sol., to appear

[3] Raniecki B., Lexcellent C. et Tanaka K., Arch. Mech. 44, 3 (1992) 261

[4] Tobushi H., Kimura K., Tanaka K., Hori T. et Sawada T., Zairyo J. of Soc. Mater. Sci., 40,457 (1991) 24

[5] Tobushi H., Iwanaga N., Tanaka K., Hori T. et Sawada T., Cont. Mech. Thermo 3 (1991) 79

[6] Kestin J. and Rice J.R., monobook corps, Stuart E.B., gal-or J. and Brainard A.J. Editeurs (1970) 275 